\title{
THERMAL CONVECTION IN A FERROMAGNETIC FLUID LAYER WITH MAGNETIC FIELD DEPENDENT VISCOSITY: A CORRECTION APPLIED
}

\author{
JYOTI PRAKASH \\ Department of Mathematics and Statistics, Himachal Pradesh University, Summer Hill, Shimla 171005, India, \\ e-mail: jpsmaths67@gmail.com \\ RAJEEV KUMAR, KULTARAN KUMARI \\ Research Scholar, Department of Mathematics and Statistics, Himachal Pradesh University, Summer Hill, Shimla 171005, India.
}

\begin{abstract}
The effect of magnetic field dependent (MFD) viscosity on thermal convection in a horizontal ferromagnetic fluid layer has been investigated numerically. A correction is applied to Sunil et al. [24] which is very important in order to predict the correct behavior of MFD viscosity. Linear stability analysis has been carried out for stationary convection. The MFD viscosity parameter $\delta$ as well as the measure of nonlinearity of magnetization $M_{3}$, both have a stabilizing effect on the system. Numerical results are also obtained for large values of magnetic parameter $M_{1}$ and predicted graphically.
\end{abstract}

Key words: ferromagnetic convection, magnetic field dependent viscosity, stationary convection, Rayleigh number

\section{INTRODUCTION}

Ferrofluids, also known as magnetic fluids, are colloidal suspensions of nanosized ferromagnetic particles stably dispersed in organic or non-organic carrier fluids, such as water, kerosene, hydrocarbon. When exposed to an external magnetic field they behave paramagnetically, with susceptibilities $\chi$ usually large for liquids [9]. Due to this property, magnetic fluids have vast applications, e.g., liquid cooled loud speakers, novel zero leakage shaft seals used in computer disc drives, energy conversion devices, liquid sealing in chemical and biochemical reactors and medical sciences [12].

Hydrodynamics of ferrofluids began with the fundamental work of Neuringer and Rosensweig [11] and the subsequent researches by Rosensweig and coresearchers, which are included later in his famous book (Rosensweig [18]). Finlayson [3] studied convective instability of a ferrofluid layer heated from below in the presence of a uniform vertical magnetic field and obtained the critical temperature gradient for the onset of instability. Lalas and Carmi [5] investigated the thermoconvective stability of ferrofluids by neglecting the buoyancy effect. Auernhammer and
Brand [1] studied the influence of rotations on the thermal convection of a magnetic fluid. For further details of investigations in ferroconvection one may be referred to Rudraiah and Shekar [19], Siddheshwar [22], Zebib [30], Vaidyanathan et al. [28], Lange [6], Suslov [26], Sunil and Mahajan [25], Prakash et al. [13], [15], [16]. The study of the thermal convection of ferrofluids in a porous medium also attracted considerable interest due to its importance in the controlled emplacement of liquids or the treatment of chemicals and the emplacement of geophysically imageable liquids into particular zones for subsequent imaging. Vaidyanathan et al. [27] investigated the thermal convection of a ferrofluid layer saturating a porous medium in the presence of a vertical magnetic field using the Brinkman model for the case of shear free boundaries. Nanjundappa et al. [10] studied the buoyancy driven ferromagnetic convection in a porous layer using the Darcy-Brinkman model with fluid viscosity different from effective viscosity. Lee and Shivakumara [7] derived the criterion for the onset of penetrative convection in a horizontal layer of a ferrofluids saturated porous medium in the presence of a magnetic field via the internal heating model. Kefayati [4] studied the heat dissipation effect of a ferrofluid on a natural convection flow in a partially 
heated cavity in the presence of an external magnetic field outside the cavity using the Lattice Boltzmann Method (LBM). Sekar et al. [20] investigated the Soret effect with regard to thermoconvective instability in a ferrofluid layer using the Brinkman and Darcy models. Mojumder et al. [8] investigated the magnetohydrodynamic convection in a half-moon shaped cavity filled with a ferrofluid.

The convection in ferrofluids gained much importance due to their astounding physical properties. One such property is the viscosity of ferrofluids. The effect of a homogeneous magnetic field on the viscosity of a fluid with solid particles possessing intrinsic magnetic moments was investigated by Shliomis [21]. Vaidyanathan et al. [29] studied the effect of MFD viscosity on ferrofluid-inducing convection in a sparsely distributed porous medium heated from below for stationary and oscillatory modes using the linear stability theory. Sunil et al. [23] also investigated the combined effect of MFD viscosity and rotation on ferrothermohaline convection saturating a porous medium in the presence of dust particles. Recently Prakash and Gupta [14] have obtained upper bounds for the complex growth rate of oscillatory motions in ferromagnetic convection with MFD viscosity in a rotating fluid layer. Prakash [16] also derived a sufficient condition for the validity of the principle of the exchange of stabilities for ferromagnetic convection with MFD viscosity in a rotating porous medium.

It is worth mentioning here that in the above cited papers on MFD viscosity these researchers carried out their analysis by considering MFD viscosity in the form $\mu=\mu_{1}(1+\vec{\delta} \cdot \vec{B})$, where $\mu_{1}$ is fluid viscosity in the absence of magnetic field $\vec{B}$ and $\vec{\delta}$ is the variation coefficient of viscosity. They decompose $\mu$ into components $\mu_{x}, \mu_{y}$ and $\mu_{z}$ which is technically not correct because $\mu$ being a scalar quantity cannot be decomposed in such a manner. Although they investigated a very important problem in ferrohydrodynamics, their results cannot be relied upon due to this wrong assumption. Recently Prakash and Bala [17] have rectified the above problem for ferromagnetic convection with MFD viscosity in a rotating sparsely distributed porous medium and derived upper bounds for the complex growth rate. Currently considerable emphasis is placed on Sunil et al. [24]. Keeping in view the above facts the basic equations are reformulated accordingly and then a mathematical and numerical analysis is carried out to rectify the weaknesses in the existing results and predict the correct solution to behavior to the problem.

\section{MATHEMATICAL FORMULATION PHYSICAL PROBLEM}

Consider the infinite horizontal layer of thickness $d$ of an electrically nonconducting Boussinesq ferromagnetic fluid confined between two boundaries $z=0$ and $z=d$, heated from below in such a way that the uniform temperature gradient $\beta=\left|\frac{d T}{d z}\right|$ is maintained (see Fig. 1). The fluid is assumed to have variable viscosity $\mu=\mu_{1}(1+\vec{\delta} \cdot \vec{B})$, where $\mu_{1}$ is the viscosity of the fluid in the absence of the applied external magnetic field. The variation of viscosity $\delta$ is assumed to be isotropic, $\delta=\delta_{1}=\delta_{2}=\delta_{3}$. Both boundaries are assumed to be free and perfect conductors of heat.

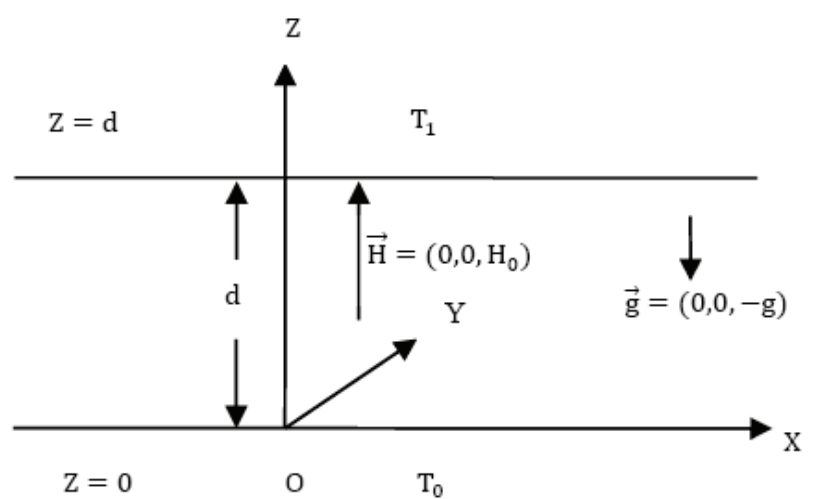

Fig. 1. Geometrical configuration

The basic governing equations for the present problem are given by [24]:

$$
\begin{gathered}
\nabla \cdot \vec{q}=0 \\
\rho_{0}\left[\frac{\partial \vec{q}}{\partial t}+\vec{q} \cdot \nabla \vec{q}\right]=-\nabla \bar{P}+\rho \vec{g}+\nabla \cdot(\vec{H} \vec{B})+\mu \nabla^{2} \vec{q} \\
{\left[\rho_{o} C_{V, H}-\mu_{O} \vec{H} \cdot\left(\frac{\partial \vec{M}}{\partial T}\right)_{V, H}\right] \frac{D T}{D t}} \\
+\mu_{O} T\left(\frac{\partial \vec{M}}{\partial T}\right)_{V, H} \cdot \frac{D \vec{H}}{D t}=K_{1} \nabla^{2} T+\phi \\
\rho=\rho_{0}\left[1+\alpha\left(T_{O}-T\right)\right]
\end{gathered}
$$

where $\vec{q}=(u, v, w), \bar{P}, \vec{H}, \mu=\mu_{1}(1+\vec{\delta} . \vec{B}), \quad \vec{g}=$ $(0,0,-g), C_{V, H}, \mu_{o}, T, \vec{M}, K_{1}, \phi, \alpha$ and $\rho$ denote, respectively, the velocity, pressure, magnetic field, variable viscosity, acceleration due to gravity, heat capacity at constant volume and magnetic field, mag- 
netic permeability, temperature, magnetization, thermal conductivity and viscous dissipation containing second order terms in velocity, the coefficient of volume expansion and density of the fluid. $\rho_{0}$ is the density of the fluid at some reference temperature $T_{O}$.

For a non-conducting fluid with no displacement current, the Maxwell's equations are given by

$$
\begin{aligned}
\nabla \cdot \vec{B} & =0, \quad \nabla \times \vec{H}=0, \\
\vec{B} & =\mu_{o}(\vec{H}+\vec{M}),
\end{aligned}
$$

It is assumed that the magnetization is aligned with the magnetic field, but a dependence on the magnitude of the magnetic field is allowed and the temperature is

$$
\vec{M}=\frac{\vec{H}}{H} M(H, T) .
$$

The linearized magnetic equation of state is

$$
M=M_{o}+\chi\left(H-H_{o}\right)-K_{2}\left(T-T_{0}\right)
$$

where $M_{o}$ is the magnetization when a magnetic field is $H_{o}$ and temperature $T_{0}, \chi=\left(\frac{\partial M}{\partial H}\right)_{H_{o}, T_{0}}$ is magnetic susceptibility and $K_{2}=-\left(\frac{\partial M}{\partial T}\right)_{H_{O}, T_{0}}$ is the pyromagnetic coefficient.

The basic state is assumed to be the quiescent state and is given by

$$
\begin{gathered}
\vec{q}=\vec{q}_{b}=0, \quad \rho=\rho_{b}(z), \quad P=P_{b}(z), \\
T=T_{b}(z)=-\beta z+T_{0}, \quad \vec{H}_{b}=\left(H_{o}-\frac{K_{2} \beta z}{1+\chi}\right) \hat{k}, \\
\vec{M}_{b}=\left(M_{o}-\frac{K_{2} \beta z}{1+\chi}\right) \hat{k}, \quad \vec{H}_{b}+\vec{M}_{b}=H_{o}+M_{o} .
\end{gathered}
$$

The Perturbed State Solutions are thus given by

$$
\begin{gathered}
\vec{q}=\vec{q}_{b}+\vec{q}, \quad \rho=\rho_{b}(z)+\rho, \quad P=P_{b}(z)+P, \\
T=T_{b}(z)+\theta, \quad \vec{H}=\vec{H}_{b}(z)+\vec{H}, \\
\vec{M}=\vec{M}_{b}(z)+\vec{M}, \quad \beta=\frac{T_{0}-T_{1}}{d} .
\end{gathered}
$$

where $\vec{q}^{\prime}=\left(u^{\prime}, v^{\prime}, w^{\prime}\right), \rho^{\prime}, P^{\prime}, \theta, \vec{H}^{\prime}$ and $\vec{M}^{\prime}$ are infinitesimal perturbations in velocity, density, pressure, temperature, magnetic field intensity and magnetization.
Substituting equation (10) into equations (1)-(8) and using equation (9), one obtains the linearized perturbation equations in the folowing form

$$
\begin{gathered}
\frac{\partial u^{\prime}}{\partial x}+\frac{\partial v^{\prime}}{\partial y}+\frac{\partial w^{\prime}}{\partial z}=0, \\
\rho_{o} \frac{\partial u^{\prime}}{\partial t}=-\frac{\partial P^{\prime}}{\partial x}+\mu_{o}\left(M_{o}+H_{o}\right) \frac{\partial H_{x}^{\prime}}{\partial z} \\
+\mu_{1}\left[1+\delta \mu_{o}\left(H_{o}+M_{o}\right)\right] \nabla^{2} u^{\prime}, \\
\rho_{o} \frac{\partial v^{\prime}}{\partial t}=-\frac{\partial P^{\prime}}{\partial y}+\mu_{o}\left(M_{o}+H_{o}\right) \frac{\partial H_{y}^{\prime}}{\partial z} \\
+\mu_{1}\left[1+\delta \mu_{o}\left(H_{o}+M_{o}\right)\right] \nabla^{2} v^{\prime}, \\
\rho_{o} \frac{\partial w^{\prime}}{\partial t}=-\frac{\partial P^{\prime}}{\partial z}+\mu_{o}\left(M_{o}+H_{o}\right) \frac{\partial H_{z}^{\prime}}{\partial z} \\
-\mu_{o} K_{2} \beta H_{z}^{\prime}+\frac{\mu_{o} K_{2}^{2} \beta \theta^{\prime}}{(1+\chi)}+\rho_{o} g \alpha \theta^{\prime} \\
+\mu_{1} \nabla^{2} w^{\prime}+\mu_{o} \mu_{1} \delta\left(M_{o}+H_{o}\right) \nabla^{2} w^{\prime}, \\
\rho_{c} \frac{\partial \theta^{\prime}}{\partial t}-\mu_{o} T_{o} K_{2} \frac{\partial}{\partial t}\left(\frac{\partial \Phi^{\prime}}{\partial z}\right) \\
=\kappa_{1} \nabla^{2} \theta^{\prime}+\left(\rho_{c} \beta-\frac{\mu_{o} K_{2}^{2} \beta}{1+\chi}\right) w^{\prime},
\end{gathered}
$$

where $\rho_{c}=\rho_{0} C_{V, H}+\mu_{0} K_{2} H_{o}$ and $\Phi$ is the perturbed magnetic potential.

$$
\begin{gathered}
H_{z}^{\prime}+M_{z}^{\prime}=(1+\chi) H_{z}^{\prime}-K_{2} \theta^{\prime}, \\
H_{i}^{\prime}+M_{i}^{\prime}=\left(1+\frac{M_{o}}{H_{o}}\right) H_{i}^{\prime}(i=1,2),
\end{gathered}
$$

where it was assumed that $K_{2} \beta d \ll(1+\chi) H_{o}$, as the analysis is restricted to physical situations, in which the magnetization induced by temperature variations is small compared to that induced by the external magnetic field.

Now $u^{\prime}$ and $v^{\prime}$ are eliminated between Eqs. (12) and (13) by operating Eq. (12) by $\frac{\partial}{\partial x}$ and (13) by $\frac{\partial}{\partial y}$, respectively, adding the resulting equations and using Eq. (11), we obtain

$$
\rho_{o} \frac{\partial}{\partial t}\left(\frac{\partial w^{\prime}}{\partial z}\right)=\left(\frac{\partial^{2}}{\partial x^{2}}+\frac{\partial^{2}}{\partial y^{2}}\right) P^{\prime}
$$




$$
\begin{gathered}
-\mu_{o}\left(H_{o}+M_{o}\right) \frac{\partial}{\partial z}\left(\frac{\partial^{2}}{\partial x^{2}}+\frac{\partial^{2}}{\partial y^{2}}\right) \Phi^{\prime} \\
-\mu_{1}\left[1+\delta \mu_{o}\left(H_{o}+M_{o}\right)\right] \nabla^{2} \frac{\partial w^{\prime}}{\partial z} .
\end{gathered}
$$

Now eliminate $P^{\prime}$ between Eqs. (14) and (17), we obtain

$$
\begin{aligned}
& \rho_{o} \frac{\partial}{\partial t} \nabla^{2} w^{\prime}=\mu_{1}\left[1+\delta \mu_{o}\left(H_{o}+M_{o}\right)\right] \frac{\partial^{2}}{\partial z^{2}}\left(\nabla^{2} w^{\prime}\right) \\
& +\mu_{1} \delta \mu_{o}\left(H_{o}+M_{o}\right) \nabla_{1}^{2}\left(\nabla^{2} w^{\prime}\right)+\mu_{1} \nabla_{1}^{2}\left(\nabla^{2} w^{\prime}\right) \\
& +\rho_{o} g \alpha \nabla_{1}^{2} \theta^{\prime}-\mu_{0} K_{2} \beta \frac{\partial}{\partial z} \nabla_{1}^{2} \phi^{\prime}+\frac{\mu_{0} K_{2}^{2} \beta \nabla_{1}^{2} \theta^{\prime}}{(1+\chi)} .
\end{aligned}
$$

where $\nabla_{1}^{2}=\left(\frac{\partial^{2}}{\partial x^{2}}+\frac{\partial^{2}}{\partial y^{2}}\right)$.

Now Eqs. (5) and (17) yield

$$
(1+\chi) \frac{\partial^{2} \phi^{\prime}}{\partial z^{2}}-\left(1+\frac{M_{o}}{H_{o}}\right) \nabla_{1}^{2} \phi^{\prime}-K_{2} \frac{\partial \theta^{\prime}}{\partial z}=0
$$

Now the perturbations $w^{\prime}, \theta^{\prime}$ and $\phi^{\prime}$, are analyzed into two dimensional periodic waves and the disturbances characterized by a particular wave number $k$ are considered. Thus it is assumed to all quantities describing the perturbation a dependence on $x, y$ and $t$ of the form

$$
\begin{gathered}
\left(w^{\prime}, \theta^{\prime}, \phi^{\prime}\right)(x, y, z, t) \\
=\left[w^{\prime \prime}(z), \theta^{\prime \prime}(z), \phi^{\prime \prime}(z)\right] \exp \left[i\left(k_{x} x+k_{y} y\right)+n t\right],
\end{gathered}
$$

where $k_{x}$ and $k_{y}$ are the wave numbers along the $x$ and $y$ directions, respectively, $k=\sqrt{k_{x}^{2}+k_{y}^{2}}$ is the resultant wave number and $n$ is constant which can be complex in general.

On using Eq. (20) in Eqs. (18), (15) and (19) and nondimensionalizing the variables by setting

$$
\begin{gathered}
z_{*}=\frac{z}{d}, \quad w_{*}=\frac{d}{v} w^{\prime \prime}, \quad D=d \frac{d}{d z}, \\
\phi_{*}=\frac{(1+\chi) K_{1} a R^{\frac{1}{2}} \phi^{\prime \prime}}{K_{2} \rho_{C} \beta v d^{2}}, \quad \sigma=\frac{n d^{2}}{v}, \\
\theta_{*}=\frac{K_{1} a R^{\frac{1}{2}}}{\rho_{C} \beta v d} \theta^{\prime \prime}, \quad v=\frac{\mu}{\rho_{0}}, \quad R=\frac{g \alpha \beta d^{4} \rho_{C}}{v K_{1}},
\end{gathered}
$$

$$
\begin{gathered}
\delta_{*}=\delta \mu_{0} H_{o}(1+\chi), \quad M_{1}=\frac{\mu_{0} K_{2}^{2} \beta}{(1+\chi) \alpha g \rho_{0}}, \\
M_{2}=\frac{\mu_{0} T_{0} K_{2}^{2}}{(1+\chi) \rho_{C}}, \quad M_{3}=\frac{1+\frac{M_{o}}{H_{o}}}{(1+\chi)}, \\
a=k d, \quad P_{r}=\frac{\mu_{c}}{K_{1}}, \quad \sigma=\frac{n d^{2}}{v},
\end{gathered}
$$

we get the following nondimensional equations (dropping the asterisks for convenience):

$$
\begin{gathered}
\left(D^{2}-a^{2}\right)\left\{\left(1+\delta M_{3}\right)\left(D^{2}-a^{2}\right)-\sigma\right\} w \\
=a R^{\frac{1}{2}}\left\{\left(1+M_{1}\right) \theta-M_{1} D \phi\right\} \\
\left(D^{2}-a^{2}-P_{r}\right) \theta+M_{2} \sigma P_{r} D \phi \\
=-\left(1-M_{2}\right) a R^{\frac{1}{2}} w \\
\left(D^{2}-a^{2}-M_{3}\right) \phi=D \theta
\end{gathered}
$$

Since, $M_{2}$ is of very small order [3], it is neglected in the subsequent analysis and thus equation (23) takes the form:

$$
\left(D^{2}-a^{2}-P_{r} \sigma\right) \theta=-a R^{\frac{1}{2}} w .
$$

The constant temperature boundaries are considered to be dynamically free. Hence the boundary conditions are:

$$
w=0=\theta=D^{2} w=D \zeta=D \phi \text { at } z=0 \text { and } z=1,
$$

(both of the boundaries are free)

In Eqs. (22)-(26), $z$ is the real independent variable such that $0 \leq z \leq 1$ represent the two boundaries. $D=\frac{d}{d z}$ is the differentiation along the vertical coordinate, $a^{2}$ is square of the wave number, $P_{r}>0$ is the Prandtl number, $R>0$ is the Rayleigh number, $M_{1}>0$ is the magnetic number which defines the ratio of magnetic forces due to temperature fluctuation to buoyant forces, $M_{3}>0$ is the measure of the nonlinearity of magnetization, $M_{2}>0$ is a non dimensional parameter which defines the ratio of thermal flux due to magnetization to magnetic flux, $\sigma=\sigma_{r}+i \sigma_{i}$ is a complex constant in general such that $\sigma_{r}$ and $\sigma_{i}$ are real constants and in consequence the dependent variables $w(z)=w_{r}(z)+i w_{i}(z), \theta(z)=\theta_{r}(z)+i \theta_{i}(z)$ and $\phi(z)=\phi_{r}(z)+i \phi_{i}(z)$ are complex valued functions of the real variable $z$ such that $w_{r}(z), w_{i}(z), \theta_{r}(z), \theta_{i}(z)$, $\phi_{r}(z)$ and $\phi_{i}(z)$ are real valued functions of the real 
variable $z$. It may be further noted that Eqs. (22)-(26) describe an eigenvalue problem for ferromagnetic convection with MFD viscosity.

\section{MATHEMATICAL ANALYSIS}

Following the analysis of [3], the exact solution satisfying the boundary conditions (26) is given by:

$$
\begin{gathered}
w=\mathrm{A} \sin \pi z, \quad \theta=\mathrm{B} \sin \pi z, \\
\phi=-\frac{\mathrm{C}}{\pi} \cos \pi z, \quad \mathrm{D} \phi=\mathrm{C} \sin \pi z,
\end{gathered}
$$

where $\mathrm{A}, \mathrm{B}, \mathrm{C}$ and $\mathrm{D}$ are constants. The substitution of above solutions in Eqs. (22), (24) and (25) yields a system of three linear homogeneous algebraic equations in the unknowns A, B, C and D. For the existence of the non-trivial solutions of this system, the determinant of the coefficients of A, B, C and D must vanish. This determinant on simplification yields:

$$
V \sigma^{2}+W \sigma+X=0,
$$

where

$$
\begin{gathered}
V=\left(\pi^{2}+a^{2} M_{3}\right) P_{r} k^{2}, \\
W=k^{4}\left(\pi^{2}+a^{2} M_{3}\right)\left[1+P_{r}\left(1+\delta M_{3}\right)\right], \\
X=k^{6}\left(\pi^{2}+a^{2} M_{3}\right)\left(1+\delta M_{3}\right) \\
-R a^{2}\left[\pi^{2}+a^{2} M_{3}\left(1+M_{1}\right)\right],
\end{gathered}
$$

and $k^{2}=\left(\pi^{2}+a^{2}\right)$.

The condition for steady convection is obtained by putting $\sigma_{i}=0$ in Eq. (28), which on simplification yields:

$$
R_{1}=\frac{(1+x)^{3}\left(1+\delta M_{3}\right)\left(1+x M_{3}\right)}{x\left(1+x M_{3}\left(1+M_{1}\right)\right)},
$$

where $R_{1}=\frac{R}{\pi^{4}}$ and $=\frac{a^{2}}{\pi^{2}}$. Relation (32) expresses the modified Rayleigh number $R_{1}$ as a function of the dimensionless wave number $x$, magnetic parameters $M_{1}$ and $M_{3}$ and MFD viscosity parameter $\delta$.

The classical results for Newtonian ferromagnetic fluids can be obtained as the limiting case of the present study by setting $\delta=0$ in Eq. (32). We receive

$$
R_{1}=\frac{(1+x)^{3}\left(1+x M_{3}\right)}{x\left(1+x M_{3}\left(1+M_{1}\right)\right)}
$$

which is the expression for the critical Rayleigh number in classical ferromagnetic Rayleigh-Bénard convection [3].
Setting $M_{3}=0$ in Eq. (33), we obtain

$$
R=\frac{\left(\pi^{2}+a^{2}\right)^{3}}{a^{2}},
$$

which expresses the Rayleigh number for ordinary fluids [2].

\section{RESULTS AND DISCUSSION}

The dispersion relation (32) is analyzed numerically by using the software scientific work place. In Fig. 1, $R_{1}$ is plotted against wave number $x$ for $M_{1}=1$ and $M_{3}=25$ and $\delta=0.01,0.03,0.05,0.07$. It is clear that MFD viscosity has a stability effect on the onset of convection as the Rayleigh number increases with the increase in the MFD viscosity parameter. In Fig. 2, $R_{1}$ is plotted against wave number $x$ for $M_{1}=1$ and $\delta=0.09$ and $M_{3}=1,2,3$ and 4 . It is clear that magnetic parameter $M_{3}$ postpones the onset of convection in the presence of MFD viscosity and thus $M_{3}$ has a stabilizing effect on the onset of convection.

Table 1. Rayleigh numbers for $\delta=0.01,0.03,0.05,0.07$

\begin{tabular}{|c|c|c|c|c|}
\hline $\begin{array}{c}\text { Wave } \\
\text { no. } \\
x\end{array}$ & $\begin{array}{c}\text { Rayleigh } \\
\text { number } R_{1} \\
(\text { for } \delta=0.01)\end{array}$ & $\begin{array}{c}\text { Rayleigh } \\
\text { number } R_{1} \\
\text { (for } \delta=0.03 \text { ) }\end{array}$ & $\begin{array}{c}\text { Rayleigh } \\
\text { number } R_{1} \\
\text { (for } \delta=0.05)\end{array}$ & $\begin{array}{c}\text { Rayleigh } \\
\text { number } R_{1} \\
\text { (for } \delta=0.07)\end{array}$ \\
\hline 1 & 5.098 & 7.1373 & 9.1765 & 11.216 \\
\hline 2 & 8.521 & 11.929 & 15.338 & 18.746 \\
\hline 3 & 13.422 & 18.79 & 24.159 & 29.528 \\
\hline 4 & 19.628 & 27.480 & 35.331 & 43.183 \\
\hline 5 & 27.108 & 37.951 & 48.794 & 59.637 \\
\hline 6 & 35.848 & 50.187 & 64.526 & 78.865 \\
\hline 7 & 45.845 & 64.182 & 82.52 & 100.86 \\
\hline 8 & 57.095 & 79.933 & 102.77 & 125.61 \\
\hline 9 & 69.598 & 97.438 & 125.28 & 153.12 \\
\hline 10 & 83.354 & 116.69 & 150.04 & 183.38 \\
\hline
\end{tabular}

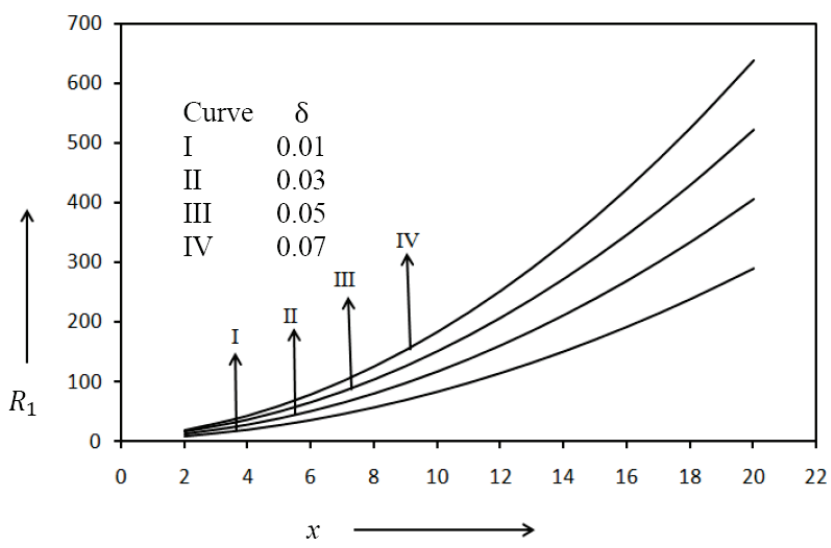

Fig. 2. Variation of Rayleigh number $\left(R_{1}\right)$, with wave number $(x)$ for $M_{1}=1, M_{3}=25, \delta=0.01,0.03,0.05$ and 0.07 for curves I, II, III and IV, respectively 
Table 2. Rayleigh numbers for $M_{3}=1,2,3,4$

\begin{tabular}{|c|c|c|c|c|}
\hline $\begin{array}{c}\text { Wave } \\
\text { no. } \\
x\end{array}$ & $\begin{array}{c}\text { Rayleigh } \\
\text { number } R_{1} \\
\left(\text { for } M_{3}=1\right)\end{array}$ & $\begin{array}{c}\text { Rayleigh } \\
\text { number } R_{1} \\
\left(\text { for } M_{3}=2\right)\end{array}$ & $\begin{array}{c}\text { Rayleigh } \\
\text { number } R_{1} \\
\left.\text { (for } M_{3}=3\right)\end{array}$ & $\begin{array}{c}\text { Rayleigh } \\
\text { number } R_{1} \\
\left.\text { (for } M_{3}=4\right)\end{array}$ \\
\hline 0.5 & 5.5181 & 5.31 & 5.3578 & 5.508 \\
\hline 1 & 5.8133 & 5.664 & 5.8057 & 6.0444 \\
\hline 1.5 & 7.0964 & 7.0238 & 7.276 & 7.6282 \\
\hline 2 & 8.829 & 8.85 & 9.2319 & 9.72 \\
\hline 2.5 & 10.905 & 11.038 & 11.571 & 12.217 \\
\hline 3 & 13.288 & 13.555 & 14.26 & 15.087 \\
\hline 3.5 & 15.963 & 16.385 & 17.284 & 18.315 \\
\hline 4 & 18.924 & 19.522 & 20.638 & 21.894 \\
\hline 4.5 & 22.165 & 22.962 & 24.316 & 25.821 \\
\hline 5 & 25.684 & 26.702 & 28.317 & 30.092 \\
\hline
\end{tabular}

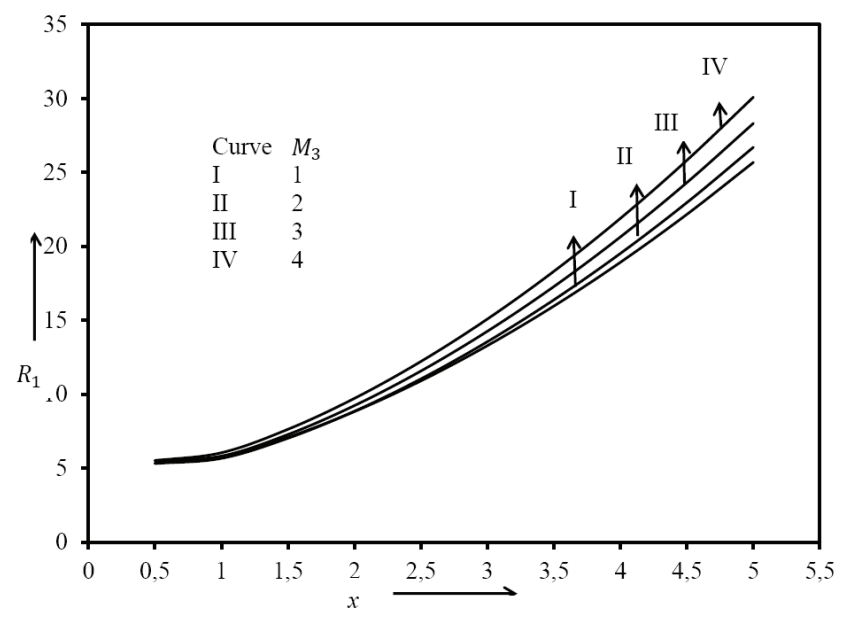

Fig. 3. Variation of Rayleigh number $\left(R_{1}\right)$

with wave number $(x)$ for $M_{1}=1, \delta=0.09$ and $M_{3}=1,2,3$, and 4 for curves I, II, III and IV, respectively

When $M_{1}$ is very large, Eq. (33) yields the results for magnetic mechanism and we get the magnetic thermal Rayleigh number $\left(=R_{1} M_{1}\right)$ for stationary mode as

$$
N=\frac{\left(1+x M_{3}\right)\left(1+\delta M_{3}\right)(1+x)^{3}}{x^{2} M_{3}} .
$$

To find the minimum value of $N$ with respect to wave number $x$, Eq. (34) is differentiated with respect to $x$ and equated to zero which resulted in the following polynomial equation:

$$
\begin{gathered}
\left(1+\delta M_{3}\right)(1+x)^{2}\left[x \left(3\left(1+x M_{3}\right)\right.\right. \\
\left.\left.+M_{3}(1+x)\right)-2(1+x)\left(1+x M_{3}\right)\right]=0 .
\end{gathered}
$$

The above equation is solved numerically by using the software scientific work place for various values of $M_{3}$ and $\delta$, and the minimum value of $x$ is obtained each time, hence critical value $N_{c}$ of $N$ is obtained.

The values of $N_{C}$ obtained in this manner for various values of $M_{3}$ and $\delta$ are given in Tables 3 and 4, respectively. The critical wave numbers characterizing the marginal state are also given. The results obtained in this table are illustrated in Figs. 4 and 5.

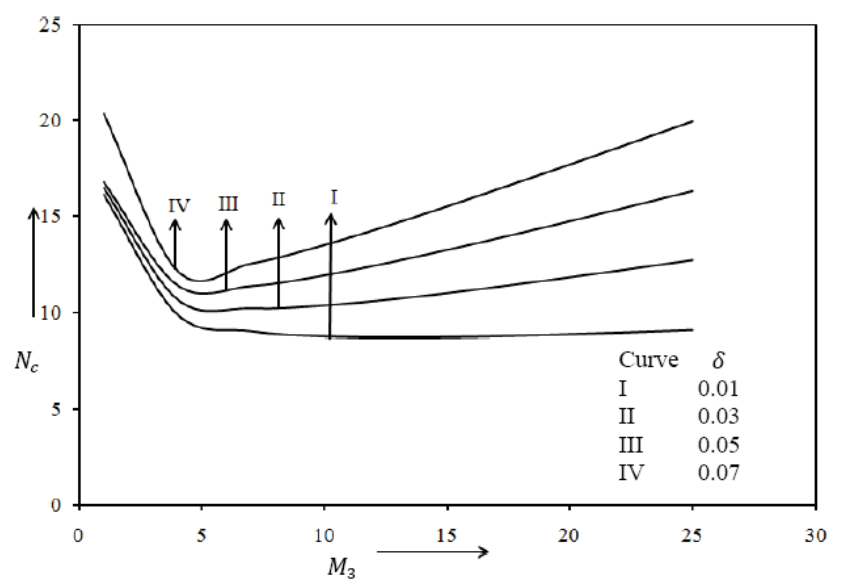

Fig. 4. Variation of critical magnetic Rayleigh number $\left(N_{c}\right)$, with the departure of linearity in the magnetic equation of state $\left(M_{3}\right)$

for $\delta=0.01,0.03,0.05$ and 0.07 for curves I, II, III and IV

Figure 4 represents the graph of $N_{c}$ versus $M_{3}$ for various values of $\delta$. Figure 4 . shows that magnetic parameter $M_{3}$ prepones the onset of convection for the smaller values of $\delta$, i.e., it has a destabilizing effect on

Table 3. Critical magnetic thermal Rayleigh numbers and wave numbers of unstable modes at marginal stability for the onset of stationary connection

\begin{tabular}{|c|c|c|c|c|c|}
\hline $\begin{array}{c}\text { Magnetization } \\
M_{3}\end{array}$ & $\begin{array}{c}\text { Critical } \\
\text { wave no. } x_{c}\end{array}$ & $\begin{array}{c}N_{c}=\left(R M_{1}\right)_{c} \\
\text { (for } \delta=0.01)\end{array}$ & $\begin{array}{c}N_{c}=\left(R M_{1}\right)_{c} \\
(\text { for } \delta=0.03)\end{array}$ & $\begin{array}{c}N_{c}=\left(R M_{1}\right)_{c} \\
(\text { for } \delta=0.05)\end{array}$ & $\begin{array}{c}N_{c}=\left(R M_{1}\right)_{c} \\
\text { (for } \delta=0.01)\end{array}$ \\
\hline 1 & 1.00 & 16.16 & 16.48 & 16.8 & 20.365 \\
\hline 4 & 0.7215 & 9.902 & 10.664 & 11.425 & 12.187 \\
\hline 7 & 0.64867 & 9.0199 & 10.2 & 11.38 & 12.56 \\
\hline 10 & 0.6131 & 8.7592 & 10.352 & 11.944 & 13.537 \\
\hline 13 & 0.59157 & 8.7024 & 10.705 & 12.707 & 14.709 \\
\hline 16 & 0.57706 & 8.7386 & 11.149 & 13.560 & 15.971 \\
\hline 19 & 0.56658 & 8.8251 & 11.643 & 14.461 & 17.279 \\
\hline 22 & 0.55864 & 8.9421 & 12.167 & 15.392 & 18.617 \\
\hline 25 & 0.55241 & 9.0788 & 12.71 & 16.342 & 19.973 \\
\hline
\end{tabular}


Table 4. Critical magnetic thermal Rayleigh numbers and wave numbers of the unstable modes at marginal stability for the onset of stationary convections

\begin{tabular}{|c|c|c|c|c|c|}
\hline $\begin{array}{c}\text { Coefficient } \\
\text { of viscosity } \delta\end{array}$ & $\begin{array}{c}\text { Critical } \\
\text { wave no. } x_{c}\end{array}$ & $\begin{array}{c}N_{c}=\left(R M_{1}\right)_{c} \\
\left.\text { (for } M_{3}=1\right)\end{array}$ & $\begin{array}{c}N_{c}=\left(R M_{1}\right)_{c} \\
\left.\text { (for } M_{3}=3\right)\end{array}$ & $\begin{array}{c}N_{c}=\left(R M_{1}\right)_{c} \\
\left.\text { (for } M_{3}=5\right)\end{array}$ & $\begin{array}{c}N_{c}=\left(R M_{1}\right)_{c} \\
\left.\text { (for } M_{3}=7\right)\end{array}$ \\
\hline 0.01 & 1.00 & 16.16 & 16.48 & 16.8 & 17.12 \\
\hline 0.03 & 0.76759 & 10.629 & 11.248 & 11.867 & 12.486 \\
\hline 0.05 & 0.68990 & 9.4742 & 10.376 & 11.279 & 12.181 \\
\hline 0.07 & 0.64877 & 9.0199 & 10.2 & 11.38 & 12.56 \\
\hline
\end{tabular}

the flow, whereas for the higher values of $\delta$ the parameter $M_{3}$ has a dual role.

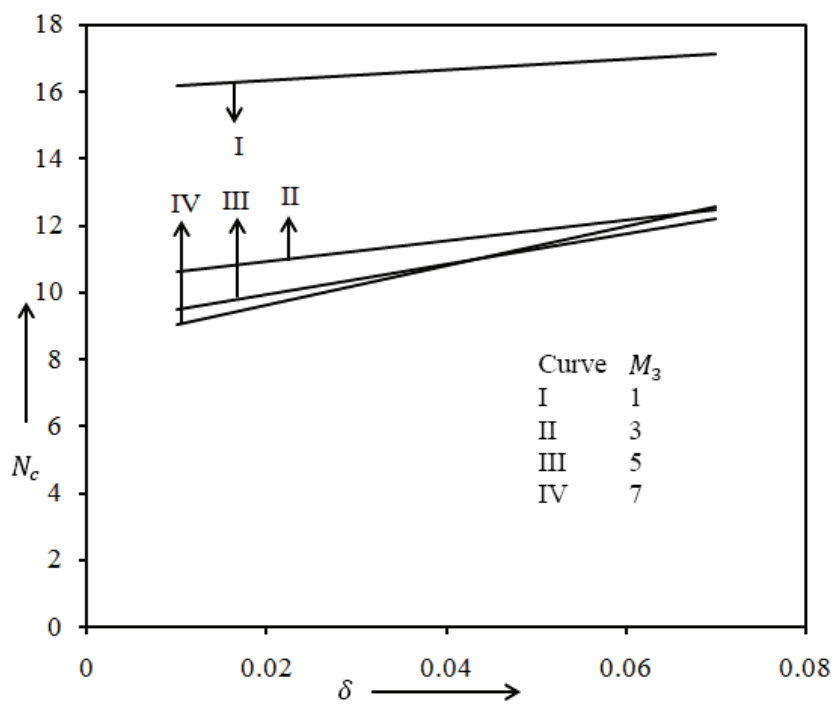

Fig. 5. Variation of critical magnetic Rayleigh number $\left(N_{c}\right)$, with magnetic field-dependent viscosity $(\delta)$ for $M_{3}=1,3,5$, and 7 for curves I, II, III and IV, respectively

Figure 5. represents the graph of $N_{c}$ versus $\delta$ for various values of $M_{3}$. This graph shows that MFD viscosity postpones the onset of convection. Thus in the present problem MFD viscosity and magnetic parameter $M_{3}$ has an opposing contribution to the stability of the system. It is worth mentioning here that the result obtained here and the result obtained by [24] (certainly using the wrong assumption regarding MFD viscosity) shows almost the same behavior but the values of critical Rayleigh number $R_{1 C}$ and critical magnetic Reynold number $N_{c}$ obtained here are definitely higher than those of [24].

\section{CONCLUSIONS}

The effect of MFD viscosity was studied on a ferromagnetic fluid layer heated from below by using linear stability analysis. A correction is applied to [24] wherein MFD viscosity $\mu=\mu_{1}(1+\delta . B)$ was com- posed of components which is otherwise not permissible as $\mu$ is a scalar quantity. Thus the results obtained in the present communication are on a correct footing and show the correct behavior of the problem. Further, the values of $R_{1 C}$ and $N_{c}$ are definitely higher than those of [24].

\section{REFERENCES}

[1] Auernhammer G.K., Brand H.R., Thermal convection in a rotating layer of a magnetic fluid, Eur. Phys. J. B, 2000, $16,157$.

[2] ChandraseKhar S., Hydrodynamic and Hydromagnetic Stability, Dover Publications, Inc., New York 1981.

[3] FInLAYSON B.A., Convective instability of ferromagnetic fluids, J. Fluid Mech., 1970, 40, 753.

[4] Kefayati G.H.R., Lattice Boltzmann simulation of natural convection in partially heated cavities utilizing kerosene/cobalt ferrofluid, IJST, Trans. Mech. Eng., 2013, 37(M2), 107.

[5] Lalas D.P., CARMI S., Thermoconvective stability of ferrofluids, Phys. Fluids, 1971, 14(2), 436.

[6] LANGE A., Thermal convection of magnetic fluids in a cylindrical geometry, J. Magn. Mag. Mater., 2002, 252, 194.

[7] LeE J., ShIVAKUMARA I.S., Onset of penetrative convection in a ferrofluid-saturated porous layer, Special Topics Rev. Porous Media: An Int. J., 2011, 2(3), 217.

[8] Mojumder S., Khan M.D.R., SAHa S., Hasan M.N., SAHA S.C., Magnetic field effect on natural convection and entropy generation in a half-moon shaped cavity with semicircular bottom heater having different ferrofluid inside, J. Magn. Mag. Mater., 2016, 407, 412.

[9] Muller H.W., Mario L., Ferrofluid Dynamics, Ferrofluids magnetically controllable fluids and their applications, Springer, 2002, 112-123.

[10] Nanjundappa C.E., ShivaKumara I.S., Ravisha M., The onset of buoyancy driven convection in a ferromagnetic fluid saturated porous medium, Meccanica, 2010, 45, 213.

[11] Neuringer J.L., RosenweIg R.E., Physics of Fluids, 7, 1927, 1964.

[12] OdenBach S., Ferrofluids: Magnetically Controllable Fluids and Their Applications, Springer, New York, 2002.

[13] PraKash J., On stationary convection and oscillatory motions in ferromagnetic convection in a ferrofluid layer, J. Magn. Mag. Mater., 2012, 324(8), 1523.

[14] PRAKASH J., GUPTA S., On arresting the complex growth rates in ferromagnetic convection with magnetic field dependent viscosity in a rotating ferrofluid layer, J. Magn. Mag. Mater., 2013, 345, 201 
[15] Prakash J., On exchange of stabilities in ferromagnetic convection in a rotating ferrofluid saturated porous layer, J. Appl. Fluid Mech., 2014, 7(1), 147.

[16] Prakash J., On the characterization of non-oscillatory motions in ferromagnetic convection with magnetic field dependent viscosity in a rotating porous medium, J. Egypt. Math. Soc., 2014, 22, 286.

[17] PRAKASH J., BALA R., On estimating the complex growth rates in ferromagnetic convection with magnetic-field-dependent viscosity in a rotating sparsely distributed porous medium, J. Appl. Mech. Tech. Phys., 2016, 57(4), 623.

[18] Rosensweig R.E., Ferrohydrodynamics, Cambridge University Press, England, 1985.

[19] Rudraiah N., SheKar G.N., Convection in magnetic fluid with internal heat generation, ASME J. Heat Transfer, 1991, $113,122$.

[20] Sekar R., RAJu K., VASAnthakUmari R., A linear analytical study on Soret-driven ferrothermohaline convection in an anisotropic porous medium, J. Magn. Mag. Mater., 2013, 331, 122.

[21] Shliomis M.I., Magnetic fluids, Soviet Phys. Uspekhi (Engl. trans.), 1974, 17(2), 153.

[22] SiddHeShwAR P.G., Rayleigh-Benard convection in a ferromagnetic fluid second sound, Jpn. Soc. Mag. Fluids, 1993, 25,32 .
[23] Sunil, Sharma A., Kumar P., Gupta U., The effect of magnetic-field-dependent viscosity and rotation on ferrothermohaline convection saturating a porous medium in the presence of dust particles, J. Geophys. Eng., 2005, 2, $238-251$

[24] Sunil, Sharma A., Sharma D., Kumar P., Effect of magnetic field dependent viscosity on thermal convection in a ferromagnetic fluid, Chem. Eng. Comm., 2008, 195, 571.

[25] SunIL, MAHAJAN A., A nonlinear stability analysis for rotating magnetized ferrofluid heated from below saturating a porous medium, Z. Angew. Math. Phys. (ZAMP), 2009, 60, 344.

[26] SUSLOV S.A., Thermomagnetic convection in a vertical layer of ferromagnetic fluid, Phys. Fluids, 2008, 20, 084101, 1.

[27] Vaidyanathan G., SeKar R., Balasubramanian R., Ferroconvective instability of fluids saturating a porous medium, Int. J. Eng. Sci., 1991, 29, 1259.

[28] Vaidyanathan G., Sekar R., Ramanathan A., Ferrothermohaline convection, J. Magn. Mag. Mater., 1997, 176, 321.

[29] Vaidyanathan G., Ramanathan A., Maruthamanikandan S., Effect of magnetic field dependent viscosity on ferroconvection in sparsely distributed porous medium, Indian J. Pure Appl. Phys., 2002, 40(3), 166.

[30] ZeBIB A., Thermal convection in a magnetic field, J. Fluid Mech., 1996, 321, 121. 